porphyria caused by a different deficiency in the liver.

The rare disorder EPP is caused by a mutation in the ferrochelatase gene. A deficiency of the ferrochelatase enzyme, which is involved in the heme biosynthesis pathway in red blood cells, leads to protoporphyrin IX build up (Nat. Biotechnol. $33,7,2015)$. Accumulated protoporphyrin reacts with light on the skin surface to induce a phototoxic reaction that can be severe.

Scenesse is a synthetic 13-amino-acid peptide analog of $\alpha$-melanocyte-stimulating hormone $(\alpha-\mathrm{MSH})$ delivered through a bioabsorbable poly(DL-lactide-co-glycolide) implant positioned under the skin. The agent binds to the melanocortin-1 receptor in dermal cells to ramp up melanin production, effectively tanning the skin. This acts as a photoprotectant, and patients taking the drug can experience the outdoors without fear of painful photosensitivity. Scenesse was already approved in the European Union in 2014, and the company published clinical trials results in 2015 in the New England Journal of Medicine; even so, it took a strong lobbying effort by patient advocates to convince the FDA that the drug had a major impact on the quality of life of people with EPP.

The data were not that compelling, says Charles Parker, hematologist at the University of Utah School of Medicine and a clinical investigator on Scenesse trials. "Patients who benefit get a big benefit, but others don't seem to get as much bronzing of the skin, so the benefit is less," he observes. Also, the endpoint in the study-tolerance to sun exposuremay have been problematic. By contrast, Mitsubishi Tanabe Pharma, which has MT-7117, a small-molecule agonist for the melanocortin receptor, in phase 2 trials for EPP, is measuring the time before participants sense a reaction to sunlight (usually itching) as the clinical endpoint. "The Mitsubishi small molecule seems to be much more uniform in the way the patients respond to it," Parker says.

The FDA could have been reticent to approve because EPP is a non-lethal disease, Parker says. But patients suffer tremendously from this condition, especially children, who can't do many of the things other children can do, he says.

Another form of porphyria, hepatic porphyria, manifests when an enzymatic deficiency in porphobilinogen deaminase affects the heme synthetic pathway in hepatocytes. In this autosomal recessive disorder, aminolevulinic acid (ALA) and porphobilinogen (PBG) build up and are released from the liver, leading to severe injury to nerves and other tissues. In the most common form, acute intermittent porphyria (AIP), attacks can include a feeling of fogginess in the head, confusion, and even epileptic fits or stroke-like syndromes and peripheral nerve injury, which predominantly manifests as recurrent severe abdominal pain. AIP, mostly affecting women between 15 and 50 years of age, is frequently confused with acute appendicitis or inflammation of the gall bladder, and those affected are often wrongly operated on. Patients may go through 5-10 years of recurrent pain before enzymatic and chemical tests correctly identify the problem.

The only sanctioned treatment for AIP is panhematin, a mixture of ironcontaining metalloporphyrin complexes derived from processed red blood cells that was approved in 1983. Although panhematin's exact mechanism of action remains to be fully understood, it is thought to work to normalize ALA and PBG by transiently suppressing levels of aminolevulinic acid synthase 1 (ALAS1), the first enzymatic step in the heme biosynthetic pathway. A failed gene therapy trial in Europe several years ago sought to treat AIP by augmenting the enzyme PBG deaminase (PGBD), halfway down the heme biosynthesis pathway. Alnylam's givosiran, a liver-targeting ligand $\mathrm{N}$-acetylgalactosamine conjugated to siRNA, inhibits the production of ALAS1 and is currently in phase 3 trials as a treatment for AIP.

"Panhematin has been a bit of a Band-Aid for patients, but chronically that drug was never able to get on top of the disease in the way patients need," says Alnylam's head of research and development, Akshay Vaishnaw. "It's better to go after ALAS1 and to suppress it using a modality like RNAi." Thus far, givosiran has reduced AIP attacks by $>74 \%$ relative to placebo, a lowering that persisted beyond the 6-month period set for the trial readout. The company submitted applications for approval of givosiran in the United States and European Union in mid-2019.

\section{Mark Ratner}

Tequesta, FL, USA

Published online: 3 December 2019 https://doi.org/10.1038/s41587-019-0347-0

\section{TB vaccine upswing for GSK}

A vaccine against Mycobacterium tuberculosis has produced good results in a phase $2 \mathrm{~b}$ trial in people with latent tuberculosis infection (TB), who represent a potential reservoir of active cases. The M72/AS01 $1_{\mathrm{E}}$ vaccine, developed by GlaxoSmithKline in partnership with the International AIDS Vaccine Initiative, provided $50 \%$ protection against progression to active pulmonary TB during 3 years after vaccination. This level of protection is in line with World Health Organization (WHO) recommendations for new TB vaccines and an improvement on previous attempts. An earlier, two-year readout of the trial, which yielded $54 \%$ efficacy, prompted the WHO to call it "unprecedented in decades of TB vaccine research".

The $\mathrm{M} 72 / \mathrm{AS} 01_{\mathrm{E}}$ vaccine is made up of an immunogenic M72 recombinant fusion protein derived from two M. tuberculosis antigens, Mtb32A and Mtb39A, with GSK's proprietary adjuvant $\mathrm{ASO}_{\mathrm{E}}$. The trial tested the vaccine on adults with a latent M. tuberculosis infection who were otherwise healthy. Overall, 3,575 HIV-negative participants from Kenya, South Africa and Zambia were enrolled. Of the 1,626 participants who received two doses of vaccine, 13 developed active pulmonary $\mathrm{TB}$, as compared with 26 (out of 1,663 participants) in the placebo group.

A vaccine against adult pulmonary TB would be a crucial tool to halt the spread of TB. The only licensed TB vaccine, BCG (bacillus CalmetteGuérin), doesn't reliably protect against the most common form of the disease, adult pulmonary TB. Other past attempts in various patient populations have also failed to make it over the $50 \%$ protection barrier: $31 \%$ efficacy with Sanofi Pasteur's adjuvanted protein subunit vaccine H4:IC3; 17\% efficacy with the viralvectored vaccine MVA85A, developed by Aeras, Wellcome Trust and OxfordEmergent Tuberculosis Consortium; $39 \%$ efficacy with Dartmouth Medical School's cell-derived $M$. vaccae vaccine, and $45 \%$ protection with BCG vaccine revaccination.

Published online: 3 December 2019 https://doi.org/10.1038/s41587-019-0350-5 